\begin{tabular}{|c|l|}
\hline Title & Dynamics of market structure driven by the degree of consumer's rationality \\
\hline Author(s) & Y anagita, Tatsuo; Onozaki, Tamotsu \\
\hline Citation & $\begin{array}{l}\text { PhysicaA: Statistical Mechanics and its A pplications, 389(5), 1041-1054 } \\
\text { https://doi.org/40.1016/.physa.2009.10.040 }\end{array}$ \\
\hline Issue Date & 2010-03-01 \\
\hline Doc URL & http://hdl.handle.net/2115/42608 \\
\hline Type & article (author version) \\
\hline File Information & PhyA 389-5_1041-1054.pdf \\
\hline
\end{tabular}

Instructions for use 


\title{
Dynamics of Market Structure Driven by the Degree of Consumer's Rationality ${ }^{\text {th }}$
}

\author{
Tatsuo Yanagita*a, Tamotsu Onozaki ${ }^{\mathrm{b}}$ \\ ${ }^{a}$ Research Institute for Electronic Science, Hokkaido University, Kita 20 Nishi 10, \\ Kita-ku, Sapporo, 001-0020, Japan \\ ${ }^{b}$ Faculty of Management and Economics, Aomori Public College, Yamazaki 153-4, \\ Goushizawa, Aomori-shi, Aomori 030-0196, Japan
}

\begin{abstract}
We study a simple model of market share dynamics with boundedly rational consumers and firms interacting with each other. As the number of consumers is large, we employ a statistical description to represent firms' distribution of consumer share, which is characterized by a single parameter representing how rationally the mass of consumers pursue higher utility. As the boundedly rational firm does not know the shape of demand function it faces, it revises production and price so as to raise its profit with the aid of a simple reinforcement learning rule. Simulation results show that 1) three phases of market structure, i.e. the uniform-share phase, the oligopolistic phase, and the monopolistic phase, appear depending upon how rational consumers are, and 2) in an oligopolistic phase, the market-share distribution of firms follows Zipf's law and the growth-rate distribution of firms follows Gibrat's law, and 3) an oligopolistic phase is the best state of market in terms of consumers' utility but brings the minimum profit to the firms because of severe competition based on the moderate rationality of consumers.
\end{abstract}

Key words: Market share dynamics, Agent-based model, Bounded rationality, Zipf's law, Gibrat's law, Oligopoly

\footnotetext{
This research was supported in part by the Japan Society for the Promotion of Science, Grant-in-Aid for Scientific Research (C), 19530155, 2007-8. The authors are grateful for their support. The authors would also like to express their gratitude to Eric Findlay for his enthusiastic and helpful comments on an earlier version.

${ }^{*}$ Corresponding author.

Email addresses: yanagita@nsc.es.hokudai.ac.jp (Tatsuo Yanagita), onozaki@bb.nebuta.ac.jp (Tamotsu Onozaki)
} 
PACS: 05.45.-a, 89.65.Gh, 89.75.-k

JEL classification: D01, D43, D83, L1

\section{Introduction}

The main purpose of this paper is to investigate the time evolution of the market structure in order to understand how oligopoly and monopoly spontaneously emerge from competition among firms. To this end, the framework of mainstream (i.e. neoclassical) microeconomics is of limited use for the reasons described below. Mainstream microeconomics claims that market structures can be classified into four categories, perfect competition, monopolistic competition, oligopoly and monopoly, according to the number of firms and the existence of product differentiation [20]. One extreme case is perfect competition, which is defined as the state of affairs where there is an indefinitely large number of firms and no product differentiation ${ }^{1}$. The other extreme case is monopoly, which is defined as the state of affairs where there is only one firm. Thus, perfect competition is on an equal footing with oligopoly and monopoly. As the term 'competition' here is prescribed mainly by largeness of the number of firms, it has a quite different meaning from what is used in economic actuality. Rather than being a state of affairs, competition in the business world is closely related to the acts of price cutting, product differentiation and so forth. The difference is not simply a matter of definition but rather, more seriously, that of the theoretical structure of mainstream microeconomics. As Hayek points out in [9], competition ought to be defined as a dynamic process through which a certain state of affairs is brought about, and not to be defined as a state of affairs per se. Price competition, for example, may result in some firms being defeated and exiting the market. In consequence, a state of affairs emerges that the total market share of surviving firms is increased. From this point of view, we can say that the dynamical property of competition is ruled out in mainstream microeconomics by the underlying premises for static analysis of a particular state of affairs, i.e. an equilibrium. In this study, we investigate the dynamic process of a 'competitive market' and one of the focuses is on describing competition as a dynamic process and studying what kind of state of affairs emerges, i.e. how market structure changes, via competition. Note that a 'competitive

\footnotetext{
${ }^{1}$ Perfect competition will be described in some more detail in Section 2.3.2.
} 
market' here is defined as a market where firms compete for better business performances such as market shares. Neoclassical microeconomics defines 'competition' as the states where there is a large number of firms. On the contrary, we define it as the acts or processes in which firms do something against rivals along the lines of Hayek. By our definition the number of firms does not matter. We need only two or more firms for competition to exist. In what follows, we focus on a competitive market where there are many firms and consumers as an initial state of market dynamics.

Another difficulty of mainstream microeconomics, which is closely related to the above, is that its paradigm is rigidly static and lacks the dynamical point of view in the true sense of the word. It supposes one-shot decision making by economic agents. Even if intertemporal decision making is considered, it is always assumed in order to ensure the rationality of agents in an uncertain world, that agents know all the future information certainly, that agents know the probability distribution of all the future states, or that agents know the true economic model and form rational expectations consistent with it. In other words, agents know the future states of the economy in advance, at least on average. In this sense, it is obvious that the time structure collapses, which mainstream microeconomics premises. It deals with a world essentially without time and not with how a market economy evolves with the passing of time. Studying the evolutionary process of a market economy does not make sense unless the premises of mainstream microeconomics are loosened and agents are accepted as boundedly rational. In this study, we investigate the time evolution of a competitive market, transforming from an initial state with many boundedly rational firms, into an oligopolistic or monopolistic state.

From a dynamical point of view, a decentralized competitive market can be regarded as a typical complex system which consists of a large number of boundedly-rational and adaptive agents interacting with each other. These micro-level local interactions give rise to a certain macro-level spontaneous order, and then, the macro-order plays the role of binding conditions for micro-behavior. For example, persons who watch and imitate others' apparel beget a fad, and then get carried away by the fad itself. Complex dynamical behavior emerges as a consequence of recurrent causal chains between individual behavior and the macro-order. This complex two-way feedback between microstructure and macrostructure has been recognized for a very long time, at least since the time of Adam Smith. Nevertheless, until recently, not only economics but other branches of science have lacked the means to model this 
feedback structure qualitatively in its full dynamical complexity. Researchers are now able to model, with the aid of high-performance computers, a wide variety of complex phenomena in a decentralized market economy, such as business cycles, endogenous trade network formation, market share fluctuations, and the open-ended coevolution of individual behavior and economic institutions. One branch of this research direction has come to be known as agent-based computational economics, i.e. computer-aided studies of the economy modeled as an evolving system of autonomous interacting agents (see, e.g. $[14,17])$.

In this study, we model a competitive market as a complex adaptive system consisting of mutually interacting, boundedly rational firms and consumers $[24,25]$. Special attention is paid to market share dynamics, in common with [18] where product differentiation exists and consumer's brand loyalty plays an important role for emerging oligopoly. In this paper, however, it is assumed that a consumer decides from which firm to purchase goods so as to increase his utility, and we employ, as a first step, a statistical description because the number of consumers is large. Aggregate consumer behavior is described by the Boltzmann distribution which is characterized by the 'inverse temperature' indicating how rationally the consumer seeks to increase his utility. A firm, on the other hand, revises production decision and price so as to raise its profit with the aid of a reinforcement learning algorithm, i.e. by learning through experience. We mainly focus on the dynamical phases which emerge as the rationality of consumers changes, and characterize their statistical properties such as the probability distribution of firms' size and growth rates.

The remainder of the paper is organized as follows. In Section 2, we present our model and demonstrate that it includes neoclassical competitive equilibria (Cournot equilibrium and perfectly competitive equilibrium) as special stationary states. In Section 3, we discuss simulation results. Firstly, an artificial monopoly case is examined in order to verify that the learning process is workable in the model. Secondly, time evolution of the competitive model is examined and it is demonstrated that all firms come to face approximately the same demand curves through a learning process. Thirdly, it is demonstrated that our model exhibits three phases, i.e. the uniform-share phase, the oligopolistic phase, and the monopolistic phase depending upon a key parameter $\beta_{1}$ which represents the degree of consumer's rationality. Fourthly, market structure dynamics is characterized from various aspects, i.e. Herfindahl index, variances, probability distributions, and averaged util- 
ity and profit. Section 4 concludes the paper.

\section{Model}

Our model consists of many consumers and many firms that are boundedly rational in the sense that they attempt to increase their utility and profit subject to information constraint and, hence, through a learning process. Consumers and firms should be considered as identical to the extent that they have the identical functions, identical behavioral rules, and identical parameters. On the other hand, they can be deemed heterogeneous in the following way. As regarding consumers, they behave according to probability and in consequence consumers as a whole follow a certain probability distribution. As for firms, the internal state which determines a firm's behavior is different from each other because its decision is affected by probabilistic learning via their past history and hence differs from firm to firm. Purchase and production decisions occur at discrete time periods. Goods are homogeneous and perishable within a unit time period.

\subsection{Consumer Behavior}

Each consumer has the same amount of money income at each time step, selects a firm, and is willing to spend all the money to purchase goods from the selected firm. For the sake of keeping the model simple, here we assume that money income at a certain period can not be carried over to the next period so as to eliminate the intertemporal allocation problem. Each consumer's utility $u_{i}(t)$ at period $t$ is represented by the identical function

$$
u_{i}(t)=U\left(x_{i}(t)\right)
$$

where $U(x)$ is a monotonically increasing function of the amount of goods $x_{i}(t)$ that a consumer obtains from firm $i$ at period $t$. $U$ is specified as $U(x)=x^{\alpha}, 0<\alpha<1$ with $U^{\prime}>0$ and $U^{\prime \prime}<0$. The consumer is willing to spend all the money to purchase as much goods as possible because his utility increases as the amount of goods increases. The amount of goods $x_{i}(t)$ he can obtain from firm $i$, however, depends not only on prices set by firm $i$ but also its output level and the number of customers who select firm $i$.

As the number of consumers is large, instead of manipulating consumer agents in fact, we employ a statistical description to represent firms' distribution of consumer share later. This description is equivalent to the result 
of simulating a model which includes a number of consumer agents acting on the following behavior rule.

Each consumer selects a firm so as to raise his utility as high as possible. He compares the utility to be obtained by purchasing goods from firm $i$ or from a randomly selected firm $j$ and selects one according to a transition probability $\rho(i, j)=\min \left(1,\left(u_{j} / u_{i}\right)^{\beta_{1}}\right)$ from firm $i$ to firm $j$ where $\beta_{1}$ is a positive parameter. This rule is interpreted as follows. If $\left(u_{j} / u_{i}\right)^{\beta_{1}} \geq 1$ (this implies $u_{j} \geq u_{i}$ ), the consumer purchases goods from firm $j$ at the next period. Furthermore, even if $\left(u_{j} / u_{i}\right)^{\beta_{1}}<1$, the consumer curiously chooses firm $j$ with a probability $\left(u_{j} / u_{i}\right)^{\beta_{1}}$, which we call the exploration probability. This rule is the so-called 'softmax action selection' in the field of reinforcement learning [22]. It implies that a firm from which consumers can purchase more goods has a higher probability of capturing consumers. The reason why exploration probability is taken into consideration is that exploring other firms might afford consumers the chance to encounter higher utility under information constraint ${ }^{2}$. Indeed, the softmax rule is used to depict the exploratory decision making of human-beings [6].

From a statistical point of view, when a consumer makes choice of a firm with such a transition probability a sufficient number of times, firm $i$ 's stationary share distribution of consumers, $w_{i}^{*}$, can be written as

$$
w_{i}^{*}(t+1)=u_{i}^{\beta_{1}}(t) / \sum_{j=1}^{M} u_{j}^{\beta_{1}}(t),
$$

where $M$ is the number of firms and the denominator is a normalization constant [4]. In other words, when we adopt the Glauber dynamics as an update rule described above, this dynamics yields an equilibrium distribution after the relaxation process. We can rewrite this share distribution as a usual form of the Boltzmann distribution: $w_{i}^{*}(t)=\exp \left(-\beta_{1} E_{i}\right) / \sum_{j} \exp \left(-\beta_{1} E_{j}\right)$, where $E_{i}=-\ln u_{i}$. Note that we can derive the multinomial logit model of boundedly rational choice in the same way as the Boltzmann distribution [15]. The multinomial logit model is originally formulated in statistics and is widely used in various fields concerning choice behavior, such as economics,

\footnotetext{
${ }^{2}$ The softmax rule is a modified version of the simplest rule called 'greedy action selection', according to which the action with highest estimated value (in our context, the firm which gives consumers highest utility) is always selected. As described later, the softmax rule indeed corresponds to the greedy rule when $\beta_{1} \rightarrow \infty$.
} 
marketing science and social psychology.

As stated above, $U$ is specified as $U(x)=x^{\alpha}, 0<\alpha<1$. In our setting, the above market share distribution can be obtained for any value of $\alpha$ as long as $\beta_{1}$ is rescaled. Thus we fix $\alpha=0.5$ without loss of generality. We note that the parameter $\beta_{1}$ corresponds to the inverse temperature in statistical mechanics [4] and represents the degree of optimization of the consumer. When $\beta_{1} \rightarrow 0$, i.e. the temperature goes to infinity (in this case, exploration probability $\left(u_{j} / u_{i}\right)^{\beta_{1}} \rightarrow 1$ ), it is interpreted that consumers behave in purely random manner irrespective of their utility, whereas all consumers select the same firm that maximizes their utility when $\beta_{1} \rightarrow \infty$, i.e. the temperature goes to zero (in this case, $\left(u_{j} / u_{i}\right)^{\beta_{1}} \rightarrow 0$ ). For these reasons, in this paper the parameter $\beta_{1}$ is characterized as the degree of rationality: the higher $\beta_{1}$, the higher the degree of rationality.

In the learning process, consumers' utility varies with time through a change in price and quantity. To take into account this effect, we introduce, for simplicity, a linear relaxation dynamics of firm $i$ 's market share, $w_{i}$, toward the stationary distribution (2) as follows:

$$
w_{i}(t+1)=w_{i}(t)-\tau\left(w_{i}(t)-w_{i}^{*}(t+1)\right),
$$

where $\tau \in[0,1]$ is a parameter that determines the relaxation time scale, or the learning rate.

\subsection{Firm Behavior}

Owing to bounded rationality, a firm does not know the demand function it faces nor the prices other firms have set, so it must decide its price $p_{i}$ and production $q_{i}$ based only on the restricted local information, i.e. changes in profits. Profit $\Pi_{i}(t)$ of firm $i$ at period $t$ is defined as

$$
\Pi_{i}(t)=p_{i}(t) s_{i}(t)-c\left(q_{i}(t)\right),
$$

where $s_{i}(t)$ denotes the quantity sold by firm $i$ and $c(q)=q^{2}$ is an identical cost function. Since functional form does not affect the results qualitatively as long as it is monotonically increasing, we employ one of the simplest forms in the present paper. An identical cost function is assumed only because we focus on an aspect of market share dynamics that is independent of the difference of cost structures. The volume of supply $s_{i}(t)$ of firm $i$ at period $t$ is represented as

$$
s_{i}(t)=\min \left(q_{i}(t), w_{i}(t) T / p_{i}(t)\right),
$$


where $T$ is the total money income of all consumers at each period. Equation (5) comes from the fact that total demand for firm $i$ 's products is given by $w_{i} T / p_{i}$, and, if the demand differs from production $q_{i}$, the supply is determined by the short-side. Thus, the profit of a firm depends upon its decision on price and production, and the demand it faces. Considering (5), the amount of goods $x_{i}(t)$ that a consumer can obtain from firm $i$ at period $t$ is written as

$$
x_{i}(t)=s_{i}(t) /\left(w_{i}(t) L\right)=\min \left(q_{i}(t) /\left(w_{i}(t) L\right), T /\left(p_{i}(t) L\right)\right),
$$

where $L$ is the number of consumers.

We assume that a firm does not directly control price and production, but instead determines rates of change of the previous price and production ${ }^{3}$. This can be regarded as a simple hedging rule in an uncertain world or rationalized by adjustment costs. Firm $i$ chooses a pair of rates of change $\left(\delta p_{i}(t), \delta q_{i}(t)\right)$ among all possible options so as to get higher profits. Note that $p_{i}(t+1)=\delta p_{i}(t) \cdot p_{i}(t)$ and similarly for $q_{i}{ }^{4}$. Here the rates of change are given by

$$
\left\{\begin{array}{l}
\delta p_{i}(t)=1+\Delta p \cos \left(2 \pi n_{i}(t) / N\right) \\
\delta q_{i}(t)=1+\Delta q \sin \left(2 \pi n_{i}(t) / N\right)
\end{array} \quad \text { for } \quad n_{i} \in\{0, \ldots, N-1\},\right.
$$

where $n_{i}(t)$ is an integer number from 0 to $N-1$ denoting a strategy of firm $i$ at period $t, N$ is the number of possible strategies, and $\Delta p$ and $\Delta q$ are given constants. Hence, the maximum rates of change in price and production are $1 \pm \Delta p$ and $1 \pm \Delta q$.

A firm selects a strategy $n \in\{0, \ldots, N-1\}$ in pursuit of higher profit, according to a simple reinforcement learning rule which is applied to the 'onearmed bandit' problem [21] as follows (here the subscript $i$ of strategy $n_{i}$ is

\footnotetext{
${ }^{3}$ Even if we assume that a firm directly controls price and production, one of the main results is preserved qualitatively that there emerges three phases of market structure depending upon $\beta_{1}$.

${ }^{4}$ Because price and production are adjusted multiplicatively, they fluctuate extensively over time. In order to avoid extensive fluctuations, maximum and minimum values of price and production are introduced. Firms cannot adjust price and production beyond the maximum values of $1.00 \times 10^{15}$ and below the minimum value of $1.00 \times 10^{-15}$. It should be noted that such maximum and minimum values do not affect the results qualitatively if they are set to be large and small enough.
} 
omitted because there is no possibility of confusion). First, a firm evaluates all of its actions $\{0, \ldots, N-1\}$ by calculating the normalized quantity

$$
\tilde{\Pi}_{i}^{n}(t)=\left(\hat{\Pi}_{i}^{n}(t)-\min _{n}\left(\hat{\Pi}_{i}^{n}(t)\right)\right) /\left(\max _{n}\left(\hat{\Pi}_{i}^{n}(t)\right)-\min _{n}\left(\hat{\Pi}_{i}^{n}(t)\right)\right),
$$

where $\hat{\Pi}_{i}^{n}(t)$ is firm $i$ 's expectation of its profit at period $t$ when it selects a strategy $n$. Then, firm $i$ selects a strategy $n$, following the 'softmax' algorithm, i.e. with a probability $\exp \left(\beta_{2} \tilde{\Pi}_{i}^{n}(t)\right) / \sum_{n=0}^{N-1} \exp \left(\beta_{2} \tilde{\Pi}_{i}^{n}(t)\right)$, where $\beta_{2}$ is the inverse temperature determining how rationally the firm behaves. Finally, firm $i$ adaptively revises its profit expectation according to

$$
\hat{\Pi}_{i}^{n}(t+1)=\hat{\Pi}_{i}^{n}(t)-k\left(\hat{\Pi}_{i}^{n}(t)-\Pi_{i}(t)\right),
$$

where $k \in[0,1]$ is the learning rate.

\subsection{Special Cases of the Model; Their Economic Implication}

For the sake of better understanding the model, we show that it includes neoclassical competitive equilibria (Cournot equilibrium and perfectly competitive equilibrium) [23] as special stationary states.

Before going on, let us begin with crucial premises of neoclassical microeconomics. Firstly, neoclassical microeconomics usually assumes perfect information; it assumes that every agent knows all the information concerning their decision making. As consumers know all the prices of firms, they select a firm which offers the lowest price. Firms know this, hence the rest of them mimic the lowest price. In this way, the 'law of indifference' (or the law of one price) holds:

$$
p_{i}=p,
$$

where $p$ is called market price. Variable $t$ is omitted here because neoclassical microeconomics is essentially a static theory, and this also applies in what follows. Secondly, neoclassical microeconomics concentrates on analyzing an equilibrium situation where supply always equals demand, so that firm $i$ 's production (or supply) $q_{i}$ also denotes firm $i$ 's demand. Thus market demand $Q$ is defined as

$$
Q=\sum_{j=1}^{M} q_{j} .
$$

Now let us define Cournot equilibrium and perfectly competitive equilibrium in a general form. 


\subsubsection{Cournot Equilibrium}

Cournot competition is a state of market in which all firms produce a homogeneous product, i.e. there is no product differentiation and they compete on the amount of output they will produce. Firms decide on their production plan so as to maximize their profits, given the competitors' amount of output. It is also assumed that market price is determined as a function of market demand:

$$
p=\mathfrak{p}(Q),
$$

which is called an 'inverse demand function'. Then firm i's profit is represented as

$$
\Pi_{i}\left(q_{i}\right)=\mathfrak{p}(Q) q_{i}-c_{i}\left(q_{i}\right) .
$$

By differentiating this with respect to $q_{i}$, we have the necessary condition for firm $i$ 's profit maximization:

$$
\mathfrak{p}^{\prime}(Q) q_{i}+\mathfrak{p}(Q)=c_{i}^{\prime}\left(q_{i}\right) .
$$

Equation (9) is called the 'reaction function' of firm $i$ and determines its production amount given the competitors' amount of output. The simultaneous solution of (9) for all $i$ is defined as a Cournot equilibrium, which is shown to exist under some reasonable assumptions by using a fixed point theorem $[7]$.

\subsubsection{Perfectly Competitive Equilibrium and the Cournot Limit Theorem}

Perfect competition is a state of market in which there are enormous numbers of small firms and all produce a homogeneous good, i.e. there is no product differentiation. Any one firm is so small relative to the whole market, just a mere drop in the ocean, that its presence or absence does not influence the market price at all. This implies that each individual firm does not have any influence on the price of the product it sells. Hence, in a perfectly competitive market, every firm is a price taker, i.e. it takes the market price as given. Firms decide on their production plan so as to maximize their profits, given the market price $p$. Then firm $i$ 's profit is represented as

$$
\Pi_{i}\left(q_{i}\right)=p q_{i}-c_{i}\left(q_{i}\right),
$$

where $p$ is a given constant. By differentiating this with respect to $q_{i}$, we have the necessary condition for profit maximization:

$$
p=c_{i}{ }^{\prime}\left(q_{i}\right) .
$$


This relationship where market price equals 'marginal cost', i.e. $c_{i}{ }^{\prime}(q)^{5}$ determines the equilibrium production amount of firm $i$ in perfect competition. The simultaneous solution of (10) for all $i$ is a perfectly competitive equilibrium, which is shown to exist under some reasonable assumptions by using a fixed point theorem [2].

\subsubsection{Neoclassical Equilibria as Special Stationary States}

Now let us move on to our model. From (5) and (8), neoclassical equality of supply and demand yields firm $i$ 's demand as

$$
q_{i}=w_{i} T / p\left(=s_{i}\right) .
$$

Thus market demand is

$$
Q=T / p
$$

In order to demonstrate that our model includes a Cournot equilibrium as a special case, by substituting (8), (11) and (12) into (4), we rewrite firm $i$ 's profit as

$$
\Pi_{i}\left(q_{i}\right)=T q_{i} / Q-c\left(q_{i}\right) .
$$

Differentiating this with respect to $q_{i}$ gives the necessary condition for profit maximization as

$$
T\left(Q-q_{i}\right) / Q^{2}=c^{\prime}\left(q_{i}\right),
$$

which corresponds to (9). Note that, as stated in Section 2.2, an identical cost function is assumed in our model only because we focus on an aspect of market share dynamics that is independent of the difference of cost structures. Next, in order to demonstrate that our model includes a perfectly competitive equilibrium as a special case, it suffices to show that the 'Cournot limit theorem' [19] holds. This theorem states that as $M \rightarrow \infty$, condition (9) gets closer to condition (10). In the context of our model, condition (13) is the counterpart of condition (9). In other words, this theorem means that the higher the number of firms, the closer a Cournot equilibrium gets to a perfectly competitive equilibrium. The left hand side of (13) is $T / Q-T q_{i} / Q^{2}=p-T q_{i} / Q^{2}$ from (12). If $M \rightarrow \infty$, then $Q \rightarrow \infty$ from (12) and the second term gets closer to zero.

\footnotetext{
${ }^{5}$ In economics, marginal cost is the change in total cost that arises when the quantity produced changes by one unit. In other words, it is the cost of producing one more unit of a good. Mathematically, the marginal cost function is denoted as the first derivative of the total cost function with respect to quantity produced.
} 
To sum up, if we additionally assume in our model $i$ ) an infinitely large number of firms, ii) perfect rationality of consumers and firms, and iii) balance of demand and supply, then the resulting stationary state is the same as perfectly competitive equilibrium of neoclassical microeconomics.

\section{Simulation Results}

\subsection{Premises}

We fix the following parameters throughout simulations: $\alpha=1.0, \tau=0.1$, $\beta_{2}=3.0, N=10, \Delta p=\Delta q=0.01, k=0.5$, and we mainly consider the dependence of consumer's behavior on the inverse temperature $\beta_{1}$, that is, on how rationally the consumer behaves. The number of firms, $M$, is also fixed during a single simulation. Our model, however, can be regarded as being equipped with a mechanism kindred to firm entry and exit because, firstly, losing all consumers owing to wrong price and/or wrong production is identical to exiting the market, and secondly, even a firm with no consumers can have implicit price and production that becomes explicit when it acquires customers and joins incumbent firms just like a new entrant. In this sense, $M$ represents the maximum number of firms that can exist in the market at the same time.

The absolute level of profit is qualitatively irrelevant to our analysis because the competition among firms drives the dynamics and only the relative volume of profit matters. Thus we set the total money income of all consumers $T$ to one, and the maximum profit of each firm is rescaled to be one. Similarly, the population $L$ of consumers is qualitatively irrelevant and is set to one.

For the initial condition of profit expectation $\hat{\Pi}_{i}^{n}(0)$, we choose an 'optimistic' value so that firms revise all their expectations effectively [21]. We set $\hat{\Pi}_{i}^{n}(0)=100 \forall i, n$, which is large enough for realizing maximum profit. The initial prices and production are $\left(p_{i}(0), q_{i}(0)\right)=(1+\xi, 1+\xi)$, where $\xi$ is a small random number distributed uniformly in $[-0.01,0.01]$. The initial market share distribution is the same among firms, i.e. $w_{i}(0)=1 / M \forall i$. As for time scale, we use a non-dimensional time $t / t^{*}$, where $t^{*}=N / k$ is a learning time scale estimated from (7).

\subsection{Artificial Monopoly Case}

In order to verify that the learning process is workable in our model, we consider the behavior of a single-firm system by setting $M=1$. This as- 


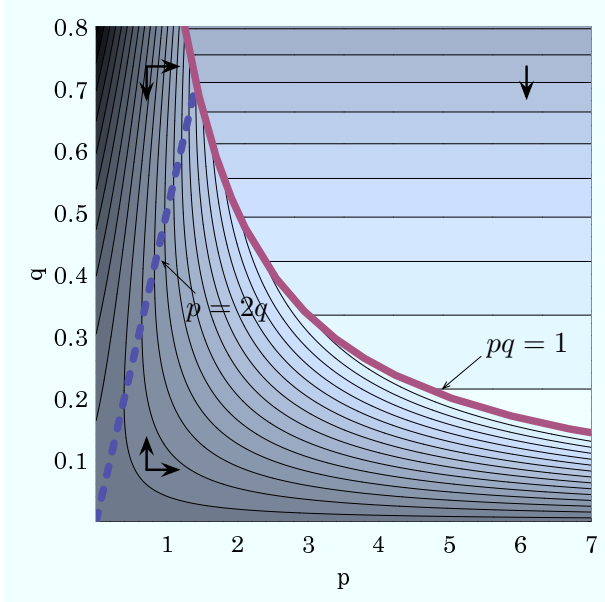

Figure 1: Gradient vector field $\nabla \Pi(p, q)$ for $M=1$ is depicted in an iso-profit contour diagram projected on the $(p, q)$-plane. The contour-lines represent a sequence of equally spaced profit values and shaded regions with higher profit values are lighter. The bold solid curve $p q=1$ is the boundary that determines which variable becomes the value of the Min function in (5). The bold dashed line $p=2 q$ corresponds to the necessary condition for $\partial \Pi / \partial q=0$.

sumption leads to a situation where monopoly is always realized artificially. Note that because there is only one firm, consumers' behavior is quite simple. Each consumer has only to go and buy from the monopolist. Hence, the dynamics of the model is entirely determined by the behavior of the monopolist. The gradient vector field $\nabla \Pi(p, q)$ is shown in Fig. 1, according to which the asymptotically best strategy of the monopolist is raising the price and reducing production along the curve $p q=1$.

The monopolist's decision histories with the learning process taken into consideration for three initial conditions in $(p, q)$-space are depicted in Fig. 2(a). The firm, even if starting from any randomly selected initial conditions, seems to update its decision through the learning process so as to increase its profit. This fact verifies that the model reproduces the profit-seeking behavior of a boundedly rational firm. The corresponding time evolution of price and production are plotted in Fig. 2(b), where the monopolist's behavior of asymptotically raising price and reducing production is observed. 
(a)

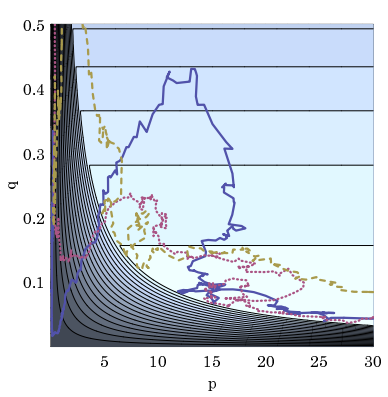

(b)

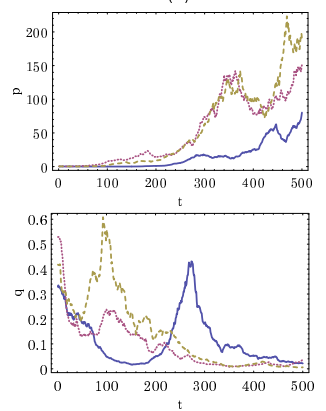

Figure 2: (a) Typical trajectories of boundedly rational, artificial monopolist's decisions for three different initial conditions are plotted in an iso-profit contour diagram projected on the $(p, q)$-plane. $t \in[0,500]$ and $M=1$. (b) Corresponding time evolutions of price and production are shown. The monopolist asymptotically raises the price and reduces production to get higher profit.

\subsection{Time Evolution with Competition}

In the artificial monopoly case discussed in the previous subsection, consumers' utility asymptotically decreases with time as a result of the firm's raising the price and reducing production. In a multi-firm system, however, simultaneously raising price and reducing production is not always the best strategy. Suppose that the market share of a particular firm is sufficiently larger than that of the others. Then the market is virtually monopolistic and the dominant firm tends to adopt the optimal strategy for the monopolist, namely raising the price and reducing production. When the dominant firm raises the price, its customers' utility decreases. The decrease in utility causes a decrease in the number of customers through (2). As a result, sooner or later through a reinforcement learning process by encountering the fact that profit is decreasing, the firm realizes that its strategy is no longer the best. The monopolist's strategy of raising the price and reducing production is restrained through feedback from consumers' behavior. With regard to production, the dominant firm slows down the pace of reducing production; otherwise its profit decreases rapidly. Typical time evolution of these values for the case where $\beta_{1}=1.0$ are shown in Fig. 3, and it is easy to confirm the above explanation from observing the movement of variables of the boldline-firm in the dotted area. The fluctuation range of prices is very wide as compared to other variables, but its absolute scale does not matter because 
it is the difference between consumers' utility that drives the dynamics.

It is worth noting here that our model implies 'the law of demand', i.e. the negative correlation between price and demand. As total demand for firm $i$ is $w_{i} T / p_{i}$, a demand curve for firm $i$ is given by plotting pairs of $\left(w_{i} T / p_{i}, p_{i}\right)$ on the plane. Demand curves for all firms are superimposed in Fig. 4, which shows that all the firms face approximately the same, well-behaved demand curves. This is because each firm comes to perceive its true demand curve after a while through a learning process.

\subsection{Market Structure Dynamics}

\subsubsection{Emerging Three Phases}

Dynamics of the market crucially depends on the parameter $\beta_{1}$, which represents how rationally consumers seek higher utility. As stated in Section 2.1, for lower rationality (smaller $\beta_{1}$ ), it is interpreted that almost all consumers choose firms in a purely random manner, i.e. $\rho(i, j) \approx 1$, so the choice is irrelevant to consumer's utility. Thus, the market share distribution is almost uniform as depicted in Fig. 5(a) and we call this case a uniformshare phase. For higher rationality (larger $\beta_{1}$ ), it is interpreted that almost all consumers choose the 'best' firm so as to seek higher utility. Hence, as shown in Fig. 5(c), monopoly emerges as a 'quasi-stationary' state, which means that strong monopolists drastically change places with time. Whereas 'quasi-stationary monopoly' is sustained, the time evolutions of the price and production are similar to those of the single-firm system. For intermediate rationality, it is interpreted that some consumers choose firms in a purely random manner and the other consumers choose the 'best' firm. As shown in Fig. 5(b), oligopoly persists owing to a balance between these two effects although severe market-share battles among oligopolists are observed.

\subsubsection{Herfindahl Index}

In order to characterize the market share dynamics, let us use the Herfindahl index $[10,12]$

$$
H(t)=\sum_{i=1}^{M}\left(w_{i}(t)\right)^{2},
$$

which measures the non-uniformity of share distribution $w_{i}$, and was first introduced in economics to indicate the concentration ratio of industries. In physics, the same index was introduced in spin glass theory [16] and is applied to complex chaotic dynamics [13]. The time evolution of $H(t)$ is 


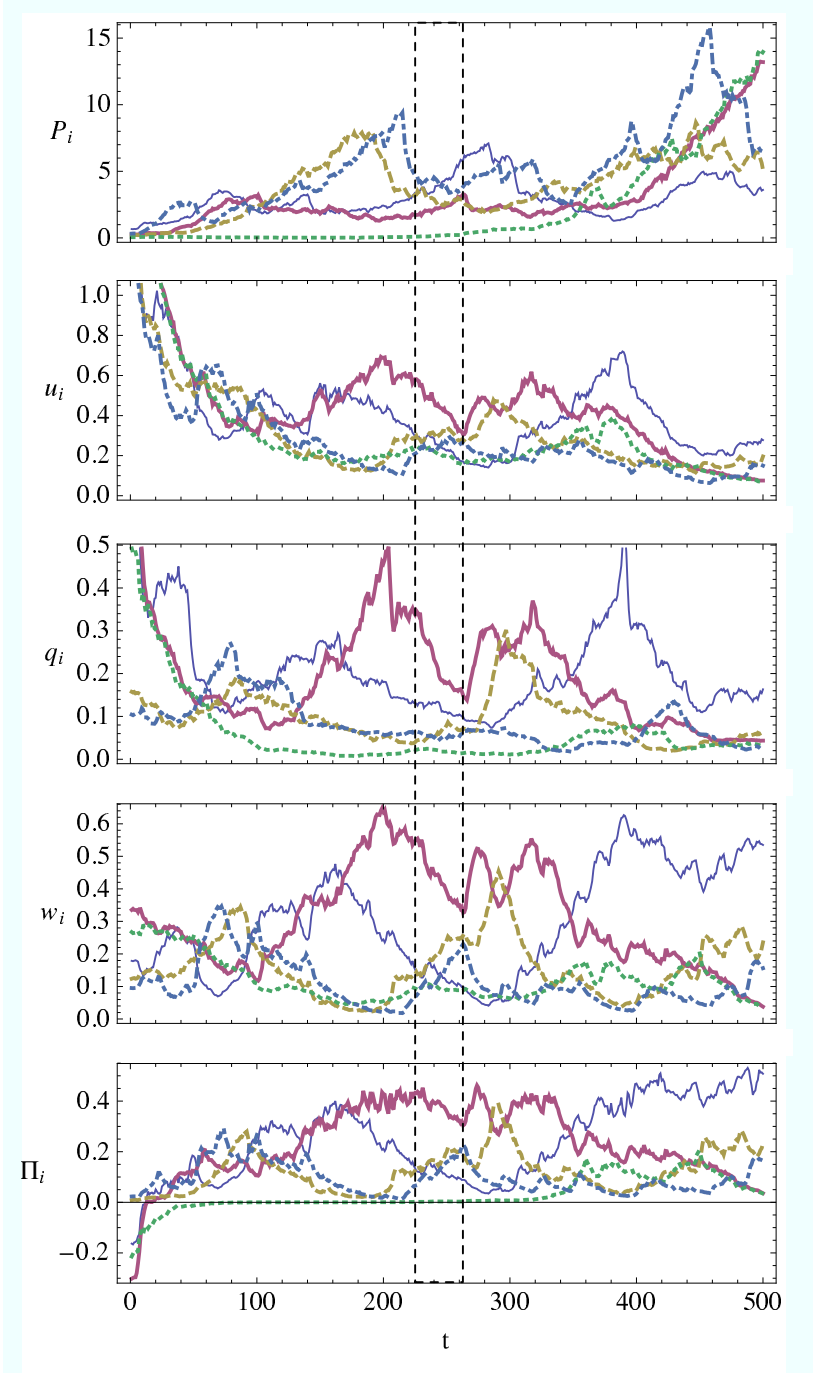

Figure 3: Typical time evolution of price $p_{i}$, utility $u_{i}$, production $q_{i}$, share $w_{i}$ and profit $\Pi_{i}$ in the five-firm system are plotted. $\beta_{1}=2.0, M=5$ and $t \in[0,500]$. 


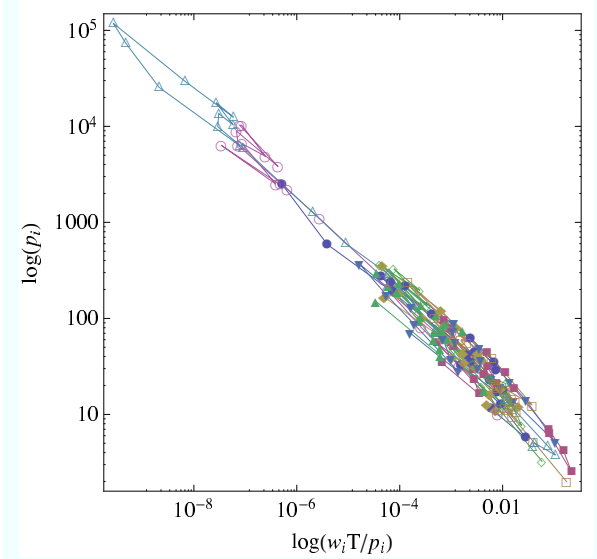

Figure 4: Demand functions for all firms are superimposed with different marks. $\beta_{1}=1.0$, $M=10$ and $t \in[500,2500]$.

(a)

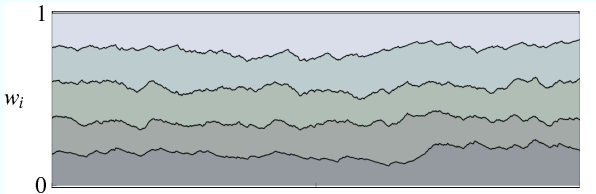

(b)

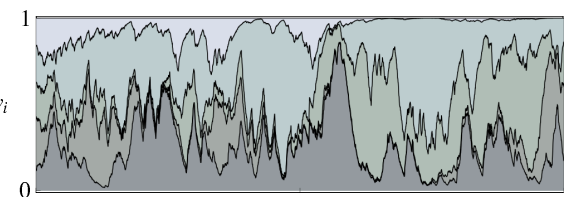

(c)

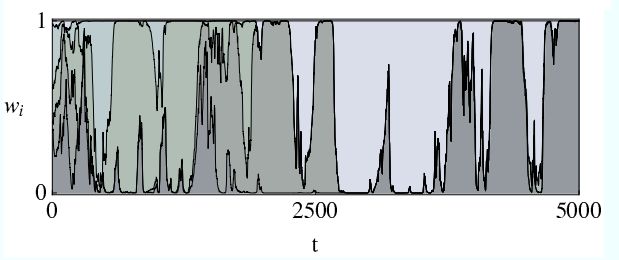

Figure 5: Time evolution of the cumulative market share in the five-firm system are shown for different values of $\beta_{1} . M=5$ and $t \in\left[0,5.0 \times 10^{3}\right]$. (a) $\beta_{1}=0.1$ : Phase of uniform share - market shares are almost uniform among firms because each consumer has low rationality. (b) $\beta_{1}=1.0$ : Phase of oligopoly market-share battle - market shares change dynamically. (c) $\beta_{1}=5.0$ : Phase of alternating monopoly - monopolistic firms alternate drastically. 
depicted in Fig. 6(a-c). Note that the index $H(t)$ ranges from $1 / M$ to $1.0^{6}$. For smaller $\beta_{1}, H(t)$ takes the values close to $1 / M$ all the time, meaning that the uniform-share state is realized because each consumer selects a firm without considering his utility. Thus, the dynamics in this case is the same as that in the artificial monopoly case: firms may set any prices and produce any amount because this does not significantly affect customers' behavior. In consequence, price gradually increases and production decreases. This case is characterized as an unnatural coexistence of multiple monopolists. For large $\beta_{1}$, consumers are very rational and over-concentration may occur in one firm that seems to give highest utility. Thus a monopolistic firm change places and $H(t)=1.0$ frequently (see Fig. 6(c)). The duration of monopoly gradually increases as $\beta_{1}$ increases. For intermediate values of $\beta_{1}$, oligopoly naturally occurs due to competition among firms through the feedback of consumers' behavior. Hence, $H(t)$ tends to take some intermediate value between $1 / M$ and 1.0. As $H(t)$ fluctuates with time, we calculate the time average of the Herfindahl index $\langle H\rangle_{t}=(1 / T) \sum_{t=1}^{T} H(t)$ in order to see its $\beta_{1}$-dependence. $\langle H\rangle_{t}$ gradually increases with $\beta_{1}$ as a consequence of the appearance of non-uniform share distribution, and approaches 1.0 corresponding to the emergence of an eternal monopolist (see Fig. 6(d)).

\subsubsection{Probability Distribution of Profit}

The transition among a uniform, an oligopoly and a monopoly market is also statistically characterized by means of the probability distributions of profit at a specific point in time $\hat{t}$. Profit of a firm mostly obeys the power law (or Pareto's law), so that

$$
P\left(\Pi_{i}\right) \propto \Pi_{i}^{-\mu} .
$$

Note that, for a small value of $\beta_{1}$ (e.g. $\beta_{1} \leq 0.1$ ), a relatively narrow range of probability distribution of profit seems to obey power law or profit may

\footnotetext{
${ }^{6}$ As the arithmetic mean $\bar{w}(t)$ of market share distribution is equal to $1 / M$, the variance $\operatorname{Var}\left(w_{i}(t)\right)$ of $w_{i}(t)$ is calculated as $\operatorname{Var}\left(w_{i}(t)\right)=1 /(M-1) \sum_{i=1}^{M}\left(w_{i}(t)-\bar{w}(t)\right)^{2}=$ $H(t) / M-1 / M^{2}$. Solving the above equation with respect to $H(t)$ yields $H(t)=$ $1 / M+(M-1) \operatorname{Var}\left(w_{i}(t)\right)$, which implies that a higher variance due to a higher level of non-uniformity of firms' shares results in a lower value of $H(t)$. The variance $\operatorname{Var}\left(w_{i}(t)\right)$ takes the lowest value of zero when consumers are equally distributed to each firm, i.e. $w_{i}(t)=1 / M \forall i$. Then the lowest value of $H(t)$ is equal to $1 / M$. When there is a firm, say $i$, that satisfies $w_{i}(t)=1$ and otherwise $w_{j}(t)=0$ for $j \neq i$, the variance $\operatorname{Var}\left(w_{i}(t)\right)$ takes the highest value of $1 / M(1-1 / M)$. Then the highest value of $H(t)$ is equal to 1 .
} 


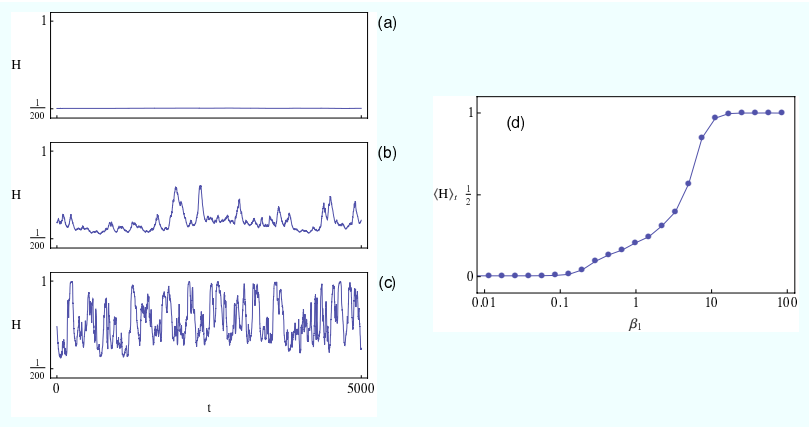

Figure 6: Left: Time evolution of the Herfindahl index $H(t)$ are shown for different values of $\beta_{1} . M=200$ and $t \in[0,5000]$. (a) $\beta_{1}=0.1$ : As market shares are uniform among firms, $H(t)$ takes the minimal value $1 / M$ almost always. (b) $\beta_{1}=1.0$ : Oligopolistic states are formed and $H(t)$ takes some certain intermediate values between $1 / M$ and 1.0. Oligopoly comes from competition among firms through the feedback of consumers' behavior. (c) $\beta_{1}=5.0$ : Monopolistic firms alternately appear and $H(t)$ takes 1.0 often. Right: (d) The time-averaged $H(t)$ vs. $\beta_{1}$ is shown for $M=200$. For smaller $\beta_{1},\langle H\rangle_{t} \approx 1 / M$, i.e. the share $w_{i}$ is uniformly distributed among firms. Beyond the critical value of $\beta_{1}^{*} \approx 0.45$, oligopolistic firms emerge and the transition of states is clearly observed. $\langle H\rangle_{t}$ gradually increases with $\beta_{1}$ and approaches 1.0. Herfindahl index is averaged over $1.0 \times 10^{4}$ time steps $\left(t \in\left[5.0 \times 10^{3}, 1.5 \times 10^{4}\right]\right)$ and sampled over 10 different initial conditions. 


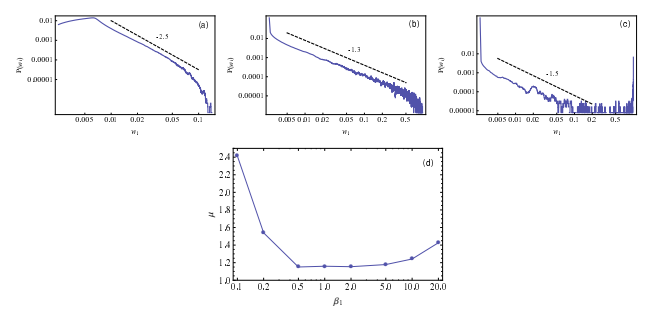

Figure 7: The probability distributions of $\Pi_{i}$ are shown. $M=100$. (a)-(c) Averaged over different $1.0 \times 10^{3}$ initial conditions. $\hat{t}=1.0 \times 10^{4}$. (a) $\beta_{1}=0.1$. (b) $\beta_{1}=1.0$. (c) $\beta_{1}=10.0$. (d) Averaged over different five initial conditions. $\hat{t}=2.0 \times 10^{4}$.

obey the exponential distribution instead. However, in order to observe the $\beta_{1}$-dependence of the power exponent, we assume that the probability distribution of profit follows a power-law relation for all levels of $\beta_{1}$. As shown in Fig. $7(\mathrm{a}-\mathrm{d})$, the profit distribution seems to obey Pareto's law with the exponent $\mu \approx 1.3$ for medium values of $\beta_{1}$, and the exponent becomes higher for smaller or larger $\beta_{1}$. Note here that in estimating $\mu$ 's we use the method proposed by [11]. A power-law relation with an exponent being approximately unity is known as Zipf's law, which is widely observed in various phenomena including firm size distribution [3, 8]. As shown in Fig. 7(d), profit distribution of our model seems to obey Zipf's law when oligopoly emerges. It should be noted that these results support the claim that the situation where the exponent of the power law is approximately unity is the transition point between the oligopoly phase and the uniform-share phase $[1,5,8]$.

\subsubsection{Probability Distribution of Firms' Growth Rate}

The transition among three phases is also statistically characterized by means of the probability distributions of firms' growth rate. The firm size measured by the volume of profit varies with time in the course of the market share dynamics. In order to characterize the underlying dynamics which governs firm size fluctuation, let us introduce the notion of logarithmic growthrate of firm's profit defined as $r_{i}=\log _{10}\left(\Pi_{i}(t+1) / \Pi_{i}(t)\right)$ and examine its probability distributions. We divide the range of $\Pi_{i}$ into logarithmically equal bins, $\Lambda_{m}=\left\{i \mid 2^{-(m+1)} \leq \Pi_{i}(t) \leq 2^{-m}\right\}(m=0, \ldots, 10)$, and then calculate the probability distributions of $r_{i}$ for each bin in order to check its statistical dependence on $\Pi_{i}$ by graphical method. The results are shown in Fig. 8. It shows that the probability distributions of $r_{i}$ have little statistical 
dependence on $\Pi_{i}$ for the case where $\beta_{1}=1.0$, because all the curves for different $m$ seem to overlap to make a single curve. This means that the growth rate is independent of firm size. The fact is often mentioned as Gibrat's law, which states that the conditional probability $P\left(r_{i} \mid \Pi_{i}(t)\right)$ is independent of $\Pi_{i}(t)$, i.e.,

$$
P\left(r_{i} \mid \Pi_{i}(t)\right)=P\left(r_{i}\right),
$$

and is mainly observed for large firms $[1,8,21]$.

\subsubsection{Averaged Utility and Profit}

Finally, we investigate the $\beta_{1}$-dependence of utility and profit. For this purpose, we calculate the time average of the ensemble mean of consumers' utility, i.e. $\langle E(u)\rangle_{t}=(1 / T) \sum_{t=1}^{T} E(u(t))$ where $E(u(t))=\sum_{i=1}^{M} w_{i}(t) u_{i}(t)$. We also calculate the time average of firms' profit per capita, i.e. $\langle\bar{\Pi}\rangle_{t}=$ $(1 / T) \sum_{t=1}^{T} \bar{\Pi}(t)$ where $\bar{\Pi}(t)=(1 / M) \sum_{i=1}^{M} \Pi_{i}(t)$.

In Fig. 9(a1) and (b1), $\langle E(u)\rangle_{t}$ and $\langle\bar{\Pi}\rangle_{t}$ are depicted as functions of $\beta_{1}$ for different numbers of firms. It is clearly seen that, for each number of firms, there is an optimal rationality value of $\beta_{1}$ at which the time-averaged consumers' utility is maximized. For smaller $\beta_{1}$, the time-averaged utility is very small: it is interpreted that each consumer selects a firm in a purely random manner (note that firm's best decision in this case is raising the price and reducing production). With the increase of $\beta_{1}$, the market-share battle in oligopoly starts to emerge whereas the time-averaged utility gradually increases and takes an optimal value that gives the maximum utility. Beyond the optimal value, the time-averaged utility gradually decreases: it is interpreted that each consumer is too rational to choose the best firm by seeking higher utility, thereby causing the formation of a monopoly market. On the other hand, the time-averaged profit per capita $\langle\bar{\Pi}\rangle_{t}$ gradually decreases with increasing $\beta_{1}$ and reaches the minimal value at a certain level of $\beta_{1}$. Beyond the minimum value, the time-averaged profit per capita gradually increases: it is interpreted that monopoly starts to emerge. In the vicinity of the optimal rationality value, oligopoly emerges although its membership changes frequently, and the time-averaged profit per capita of oligopolistic firms reaches the minimal value. Oligopoly is the best state of the market in terms of consumers' utility whereas it brings the minimal profit to participants because of severe competition. Note that, as shown in Fig. 9(a2) and (b2), the rationality value of $\beta_{1}$ which maximizes consumers' utility or minimizes firms' profit varies as the number of firms increases and converges to a certain limit value. 

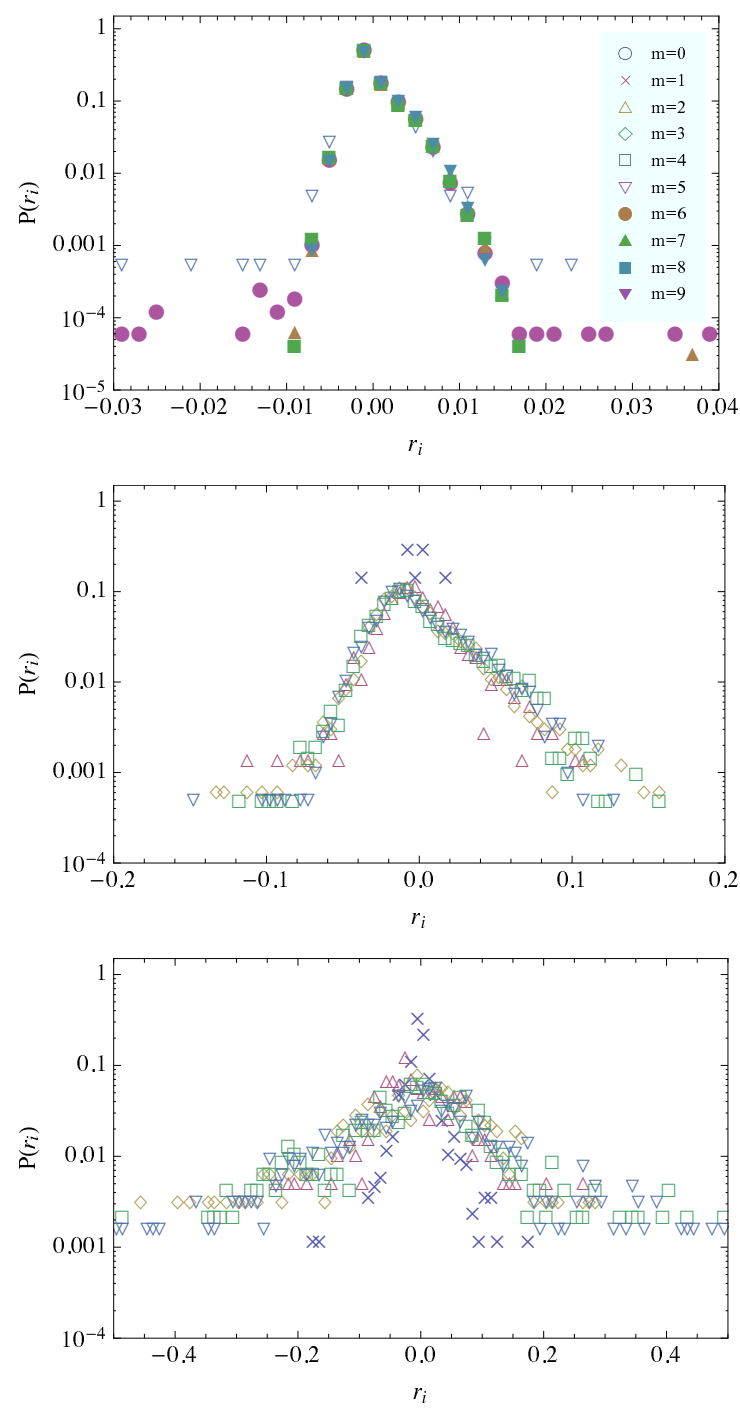

Figure 8: The probability distributions of logarithmic growth rate $r_{i}$ for $M=100$ are shown. The range of $\Pi_{i}$ is divided into logarithmically equal bins, $\Lambda_{m}=\left\{i \mid 2^{-(m+1)} \leq\right.$ $\left.\Pi_{i}(t) \leq 2^{-m}\right\}(m=0, \ldots, 9)$, and the probability distributions of $r_{i}$ are calculated for each bin. The growth rate seems to be independent of firm size for (b). (a) $\beta_{1}=0.1$. (b) $\beta_{1}=1.0$. (c) $\beta_{1}=5.0$. 

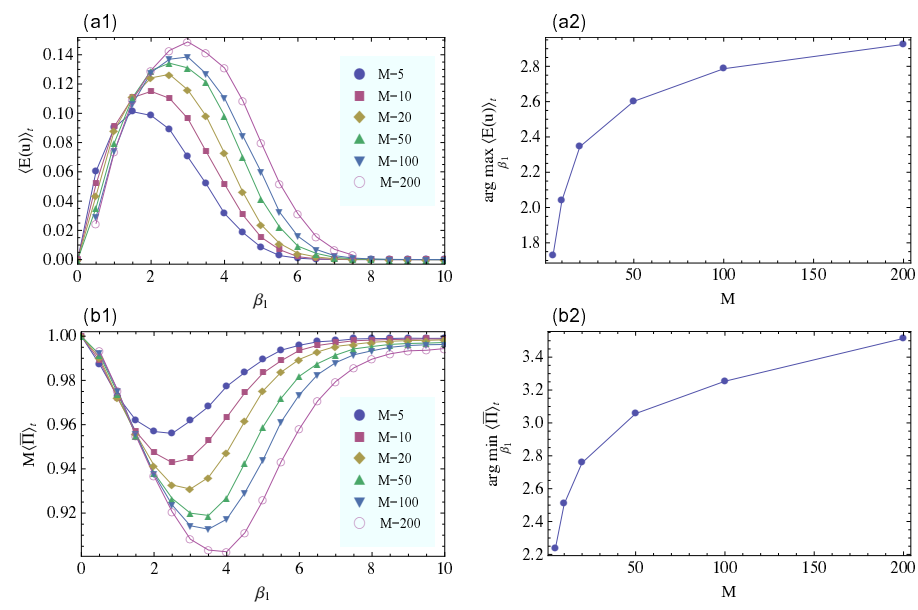

Figure 9: (a1) The time average of the mean of consumers' utility $\langle E(u)\rangle_{t}$ and (b1) the time average of firms' profit per capita $\langle\bar{\Pi}\rangle_{t}$ versus $\beta_{1}$ are plotted for $M=5,10,20,50,100$ and 200. The utility and profit are averaged over $1.0 \times 10^{5}$ time steps $\left(t \in\left[2.5 \times 10^{4}, 1.25 \times 10^{5}\right]\right)$ and sampled over 50 different initial conditions. There is an optimal rationality value which maximizes the time-averaged utility for each $M$ and there is also a rationality value which minimizes the time-averaged profit per capita for each $M$. (a2) The rationality value which maximizes the time-averaged utility and (b2) the rationality value which minimizes the time-averaged profit per capita converge to certain finite values. 


\section{Concluding Remarks}

We have investigated the dynamics of a competitive market consisting of mutually interacting, boundedly rational firms and consumers. Instead of relying on demand functions, the behavior of consumers is described by the market share distribution, i.e. the stationary distribution of a large number of consumers who are interpreted to employ softmax strategy. This distribution is characterized by a single parameter $\beta_{1}$ that represents how rationally consumers behave. Firms revise their production decisions and prices so as to raise their profit with the aid of a simple reinforcement learning rule. Numerical simulations show the following results:

i) Three phases of market structure: the uniform-share phase, the oligopolistic phase, and the monopolistic phase appear depending upon a key parameter $\beta_{1}$.

ii) In an oligopolistic phase, the market-share distribution of firms follows Zipf's law and the growth-rate distribution of firms follows Gibrat's law.

iii) An oligopolistic phase is the best state of market in terms of consumers' utility whereas oligopoly brings the minimal profit to the firms because of severe competition based on the moderate rationality of consumers.

It should be noted that the third result presents a view different from mainstream microeconomics. Neoclassical microeconomics claims that a competitive market is the most efficient in terms of both consumer's utility and firm's profit, and such efficiency is disturbed in an oligopolistic or monopolistic market. In the context of our model, an oligopolistic phase is the most efficient in terms of consumer's utility, although it is the most inefficient in terms of firm's profit.

The model proposed in this paper is a very simple one and could be developed in a number of directions. For example, firstly, because which phase appears in the market depends upon a key parameter $\beta_{1}$, it would be important to endogenize $\beta_{1}$. Secondly, it would be of interest to introduce network structures into consumers to represent information exchanges or into firms to represent trade relations. Third, a more realistic framework should consider various strategies of firms such as product differentiation, advertising, and so forth. 


\section{References}

[1] Aoyama H et al (2007): Pareto Firms. Nippon Keizai Hyoron-sha, Tokyo (in Japanese)

[2] Arrow KJ, Debreu G (1954): Existence of an Equilibrium for a Competitive Economy. Econometrica 22: 265-290

[3] Axtell R (2001): Zipf Distribution of U.S. Firm Sizes. Science 293: 181820

[4] Binder K, Heermann DW (1979): Monte Carlo Methods in Statistical Physics. Springer, Berlin

[5] Bouchaud J-P, Mèzard M (2000): Wealth Condensation in a Simple Model of Economy. Physica A 282: 536-45

[6] Daw ND et al (2006): Cortical Substrates for Exploratory Decisions in Humans. Nature 441: 876-9

[7] Frank CRJr, Quandt RE (1963): On the Existence of Cournot Equilibrium. International Economic Review 4: 92-96

[8] Fujiwara Y et al (2004): Do Pareto-Zipf and Gibrat Laws Hold True? Physica A 335: 197-216

[9] Hayek FA (1948): The Meaning of Competition. In: Individualism and Economic Order: 92-106. University of Chicago Press, Chicago

[10] Herfindahl OC (1950): Concentration in the Steel Industry. PhD dissertation, Columbia University, New York

[11] Hill BM (1975): A Simple General Approach to Inference about the Tail of a Distribution. Annals of Statistics 3: 1163-74

[12] Hirschman AO (1964): The Paternity of an Index. American Economic Review 54: 761-2

[13] Kaneko K (1991): Partition Complexity in a Network of Chaotic Elements. Journal of Physics A: Mathematical and General 24: 2107-19

[14] Kirman A, Zimmermann J-B (eds) (2001): Economics with Heterogeneous Interacting Agents. Springer, Berlin 
[15] Marsili M (1999): On the Multinomial Logit Model. Physica A 269: 9-15

[16] Mezard M et al (1987): Spin Glass Theory and Beyond. World Scientific, Singapore

[17] Namatame A et al (eds) (2006): The Complex Networks of Economic Interactions: Essays in Agent-Based Economics and Econophysics. Springer, Berlin

[18] Onozaki T, Yanagita T (2003): Monopoly, Oligopoly and the Invisible Hand. Chaos Solitons and Fractals 18: 537-47

[19] Ruffin RJ (1971): Cournot Oligopoly and Competitive Behavior. Review of Economic Studies 38: 493-502

[20] Stiglitz JE, Walsh CE (2006): Economics. W.W. Norton, New York

[21] Sutton J (1997): Gibrat's Legacy. Journal of Economic Literature 35: $40-59$

[22] Sutton RS, Barto AG (1998): Reinforcement Learning: An Introduction. MIT Press, Cambridge MA

[23] Varian HR (2005): Intermediate Microeconomics: A Modern Approach, rth edition. W. W. Norton, New York

[24] Yanagita T, Onozaki T (2008): Dynamics of a Market with Heterogeneous Learning Agents. Journal of Economic Interaction and Coordination 3:107-18. doi: 10.1007/s11403-008-0038-2

[25] Yanagita T, Onozaki T (2009): A Model of Market Structure Dynamics with Boundedly Rational Agents. In T. Terano et al. (Eds.): AgentBased Approaches in Economics and Social Complex Systems V: 25566. Springer, Tokyo 